Briginal ârticlea.

\section{THE RESULTS OF ONE HUNDRED AND SIXTY-EIGHT OPERATIONS FOR HERNIA.}

BY CHARLes L. SCUDDER, M.D.,

Surgeon to the Massachusetts General Hospital; Lecturer on Surgery, Harvard Medical School, Boston, Mass.

I WISH to describe my interpretation of the Bassini operation for inguinal hernia; to record the results of operations for inguinal hernia by this method; to report conditions existing following operations for the repair of other forms of hernia; to state certain important facts concerning hernia. with its fibers and freed by blunt gauze dissection from the parts beneath for about two fingers' breadths from the cut edges. The lower border of the internal oblique muscle, the sac of the hernia, the cord covered in part by the cremaster muscle and fascia are thus exposed to view. (Fig. 2.) The cremaster muscle may be well developed, as is indicated in the drawing, or it may be poorly developed and appear as a thin fascia with a few muscular fibers scattered through it. When the muscle is well developed, it may be utilized in closing the abdominal wound (Halstead), as is here illustrated.

The cremaster is split parallel with its fibers and separated from off the sac and cord and the sac developed by blunt dissection with fingers

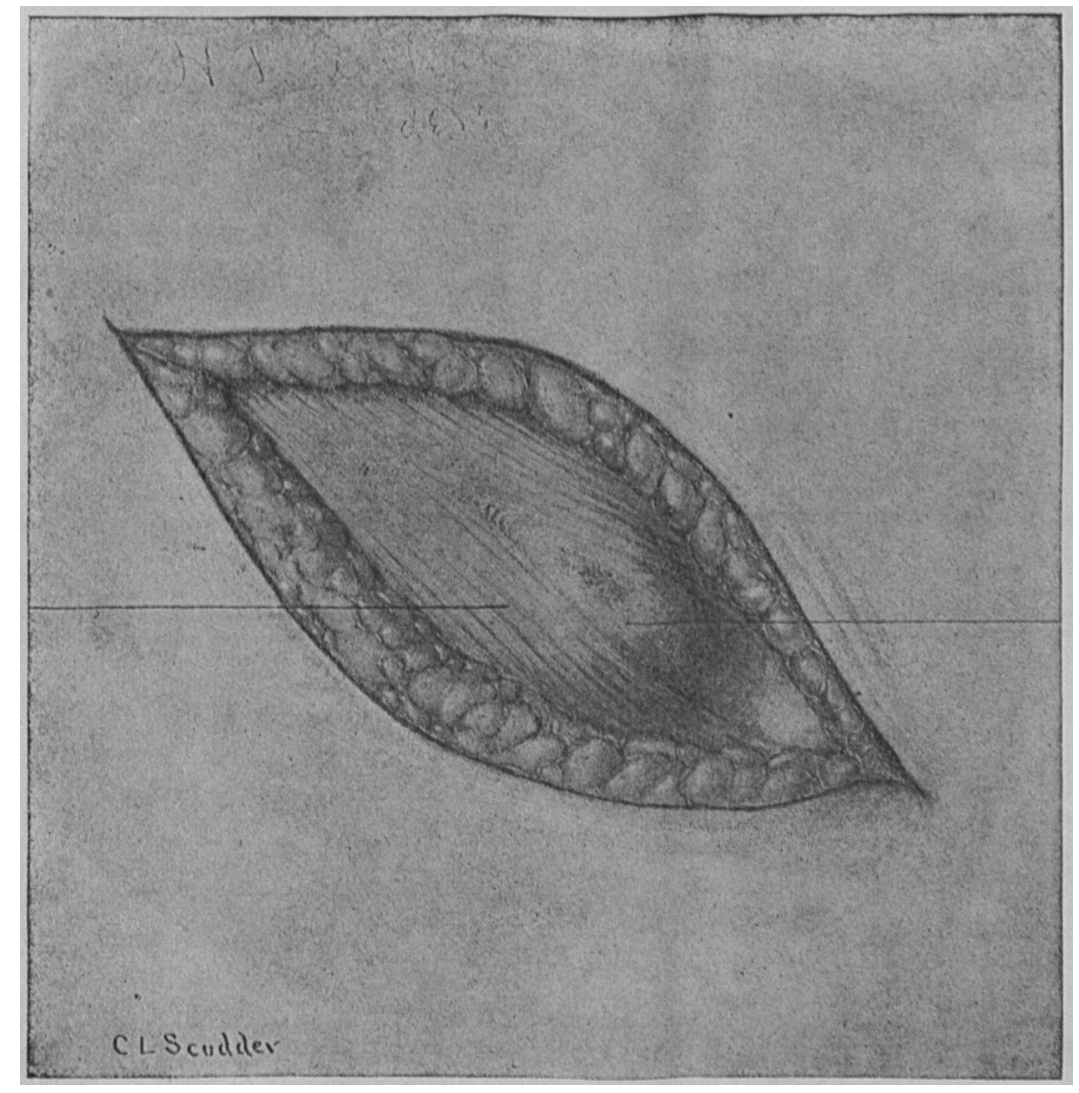

Fro. 1. Oblique incision through skin and superficial fascia down to the fascia of the externa Fro. 1. Oblique incision through skin and superficial fascis down to the fascia of the external
oblique muscle. Note the external abdominal ring made apparent by slight bulging caused by full hernial sac.

The accompanying drawings were made for and gauze. The sac lies anteriorly to the cord me from studies of the anatomical findings at and may be found high up near the situation of operations for hernia. These drawings illustrate the essential details of Bassini's operation for inguinal hernia. The operation is as follows:

A straight and obliquely placed incision is made parallel to and one finger's breadth above Poupart's ligament sufficient in length to expose readily the position of the internal and external abdominal rings. The few bleeding vessels are clamped and immediately ligated. The external abdominal ring is exactly developed by a few strokes of the knife and blunt dissection. (Fig. 1.)

The external oblique fascia is divided parallel should be carefully freed and all bleeding checked. 
If the sac is a thin and short one, not extending to the bottom of the scrotum, after it is opened, upon introducing the finger within it, the sac may readily be separated from the surrounding tissues of the cord and isolated. If the sac cannot be thus readily stripped from the surrounding parts, then after opening the sac, as shown in the drawing, it is divided transversely down to the cord and its vessels. The line of posterior division of the sac over the cord is indicated in Fig. 4. After dividing the peritoneum, the separation of the cord from the sac distal portion of the sac [the scrotal part, if such exists (Fig. 5)] it is well to remove. If it is left in situ, no suture is taken in its mouth. The cavity of this sac is probably completely obliterated. If the distal portion of the sac is undisturbed, its interior should be thoroughly wiped with gauze to facilitate its obliteration by the process of repair. I have never seen a hydrocele or cyst follow this method of treating the undissected distal portion of a thin hernial sac.

Traction downward upon the proximal portion of the hernial sac and retraction of the tissues

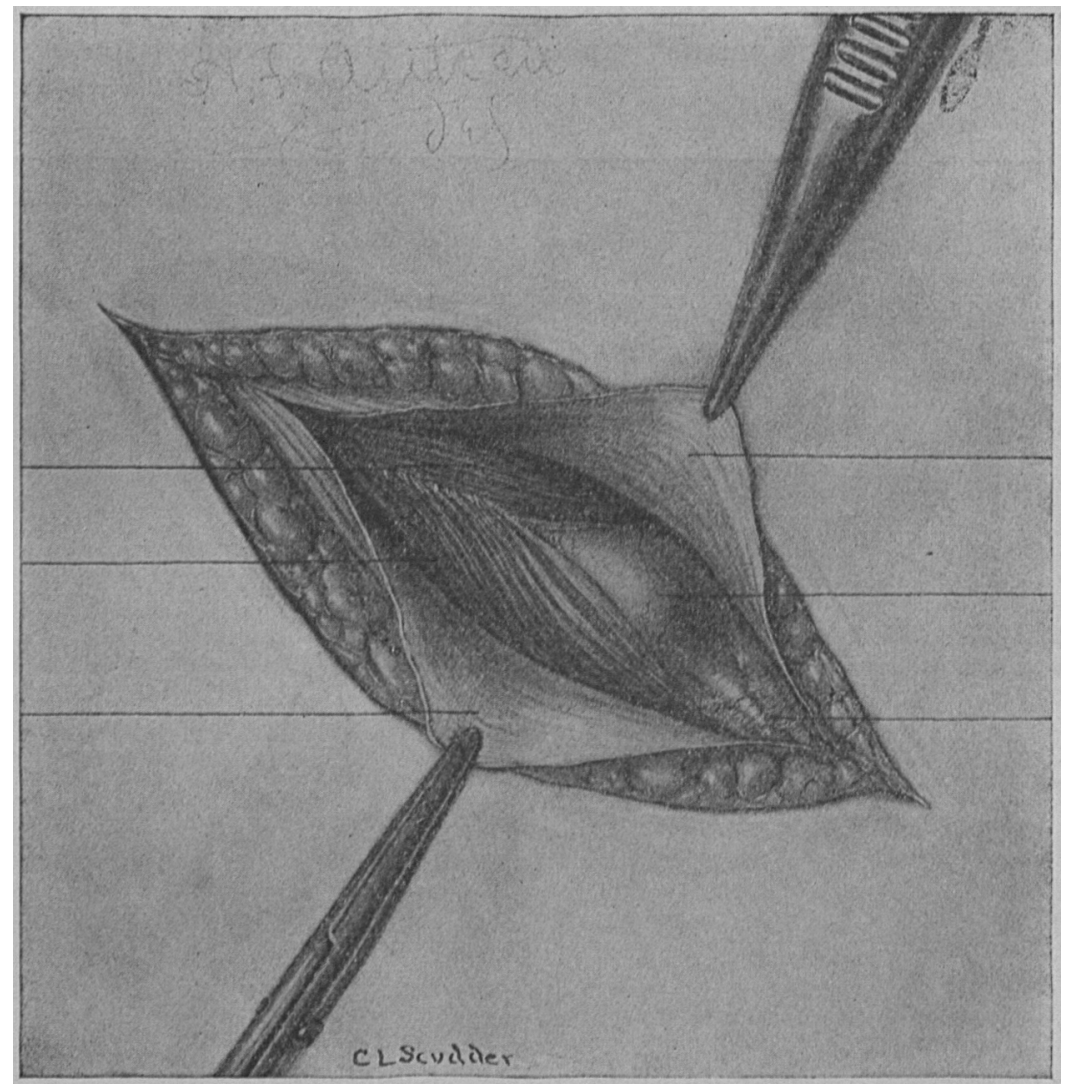

Fra. 2. Oblique incision in line of fibers of the external oblique fuscia. External oblique fascia freed from parts beneath. Note fibers above of internal oblique, conjoined tendon, below

is very greatly facilitated by blunt sponge dissection. The proximal part of the sac is isolated (a) from the cord and (b) from the abdominal muscles (the internal oblique, conjoined tendon) in order to facilitate the placing of the peritoneal suture. It is important to make the isolation of the neck of the sac complete in its whole circumference so that the subscquent suture will be an effectively placed one.

The commonly used purse-string suture, even if applied with transfixion of the neck of the sac, may slip. This occurred to me some years ago. It unfortunately happened before the outer wound was closed, so that a secure suture was immediately placed. Since that time I have used the suture illustrated in Fig. 6. When the sac is very thin walled, a purse-string suture securely applied will serve every purpose. The the (internal oblique, cremaster, etc.) at the upper angle of the wound, together with traction downward upon the cord, facilitate suturing the peritoneum. (Fig. 6.) I have used the continuous loop or hemstitching suture taken with No. 1 chromic gut and a curved needle. (Fig. 6.) The cord and distal portion of the sac, if left, are now lifted by blunt dissection from their bed, if it is decided to transplant the cord, and are together held aside. (Fig. 7.)

The next sutures are placed as indicated in Fig. 7. It is well to avoid including within the suture the ilio-inguinal and the genital branch of the genito-crural nerves below the lower border of the internal oblique. I always place one or two sutures external to the spot where the cord comes through the peritoneum (Fig. 7) and superficial to the suture of the neck of the sac. These two sutures 
preclude the possibility of a recurrence of the I think no especial dressing of a wound of hernia at this particular place. The remaining the kind described above has any advantages sutures are placed so as to lic beneath the up- over the simple dry sterile gauze. I have seen lifted cord. These interrupted sutures include used, silver foil, ointments, pastes and powders. the lower border of the internal oblique and I think that the two factors, other things being conjoined tendon, the cremaster muscle and equal, which assist or favor the healing of a fascia and Poupart's ligament. (Fig. 7.) The hernial wound are absolute hemostasis and a sutures are tied securely without constricting minimum of operative trauma (by traction, etc.) too severely the muscular tissues. (Fig. 8.) to the skin edges and to the other tissues of the

The cord is placed upon the tissues approxi- wound. Trauma to the skin may be avoided mated by this series of sutures, and the external by an ample cutaneous incision.

oblique fascia is closed over the cord by a con- So important is absolute hemostasis that I tinuous chromic catgut suture, taken as illus- not only ligate every tiny bleeding point in the trated in the drawing (Fig. 9), with a loop or wound, but I apply a ham splint to the leg of hemstitch. 'The fascia is thus not only approxi- the operated side, that the spica bandage apmated, but there is a definite overlapping or plied to hold the dressing may exert a maximum

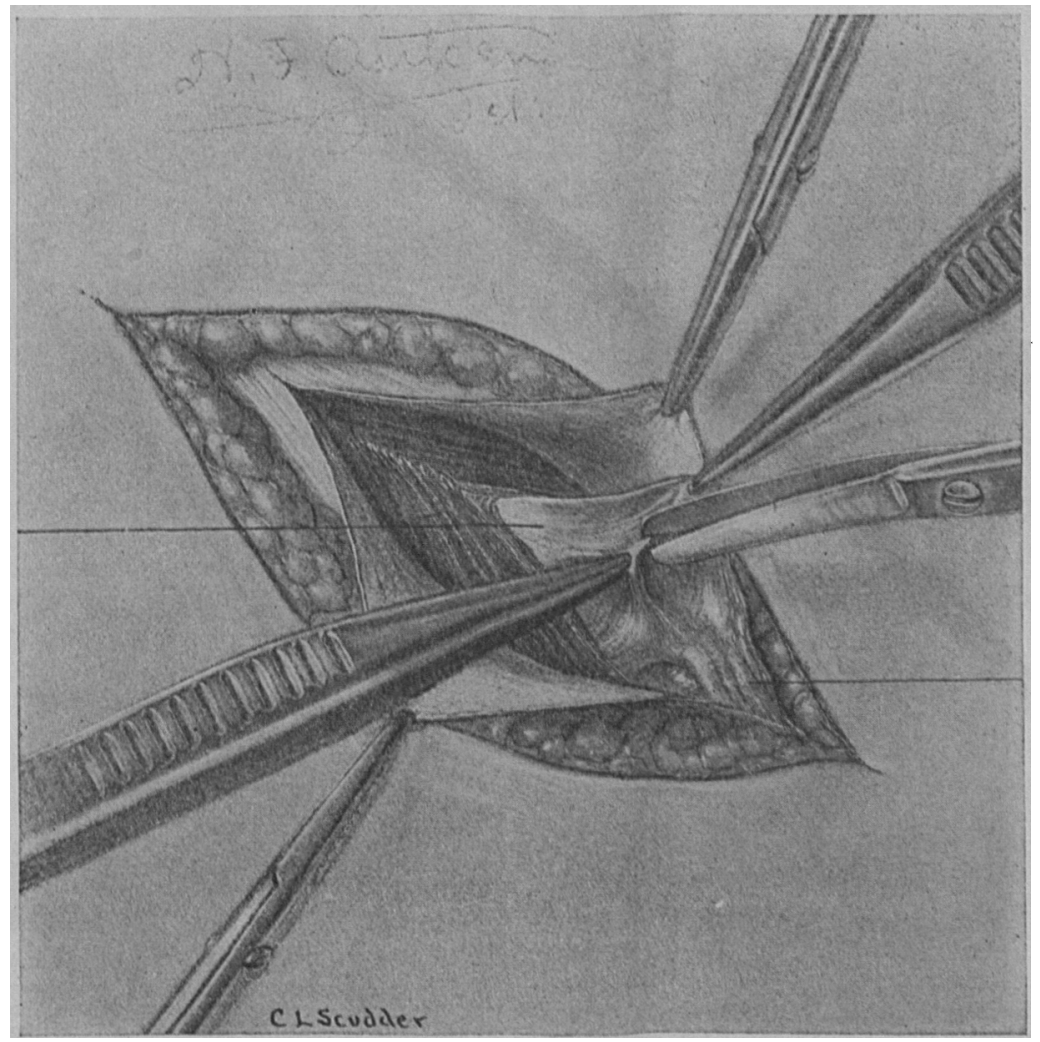

FIg. 3. The sac of the hernia has been isolated sufficiently and raised by forceps. Note scissors opening the sac, cord in lower angle of wound.

coaptation of the two layers of fascia as by the of pressure upon the wound during the first mattress suture. Mayo has called attention to twelve or twenty-four hours. The splint is the security of the mattress suture employed in then removed. There is no pressure exerted the transversely overlapped fascìa in umbilical upon the region of the groin by a spica bandage hernia. Halstead has emphasized the firmness of if the patient is permitted to flex the knee of the a wound closed by the mattress suture in cases bandaged side. A broad band of adhesive of inguinal hernia.

The superficial fascia is next approximated, and with it the subcutaneous fat tissue, by interrupted fine No. 0 catgut sutures. The skin is closed by silkworm gut, either cutaneously or subcutaneously placed. All blood and moisture are carefully wiped from the suture creases and skin. A dry sterile gauze dressing fastened with collodion is applied. plaster is used by some operators to secure the dressing and maintain pressure.

In enumerating the facts which seem to me important in the operation for the cure of an inguinal hernia, I would include the following: An ample cutaneous incision; absolute hemostasis; an anatomical dissection of the parts concerned, so that every structure is clearly recognized; the complete isolation of the neck 
or abdominal portion of the sac; the complete umbilical. These 10 deaths make a mortality emptying of the sac of both adherent and non- of $40 \%$ in the strangulated group.

adherent contents; the suture of the hernial I have succeeded in obtaining the present consac at the level of the peritoneum of the anterior dition of 158 of these cases. Of the 158 operaabdominal wall; the exact suture of the abdomi- tions, 143 wounds are solid and without renal wall superficial to the peritoneum, including currence. That is, $90.6 \%$ of the cases operated always one or two sutures placed over and above upon in whom the returns were known were the sutured sac; the minimum of trauma to the cured. cord.

In all ordinary cases of inguinal hernia, I have found this method of exposure and suture of the hernial sac and the method here described of known recurred. That is, $90.9 \%$ of the inrepair of the abdominal wall satisfactory. I guinal group were known to be cured.

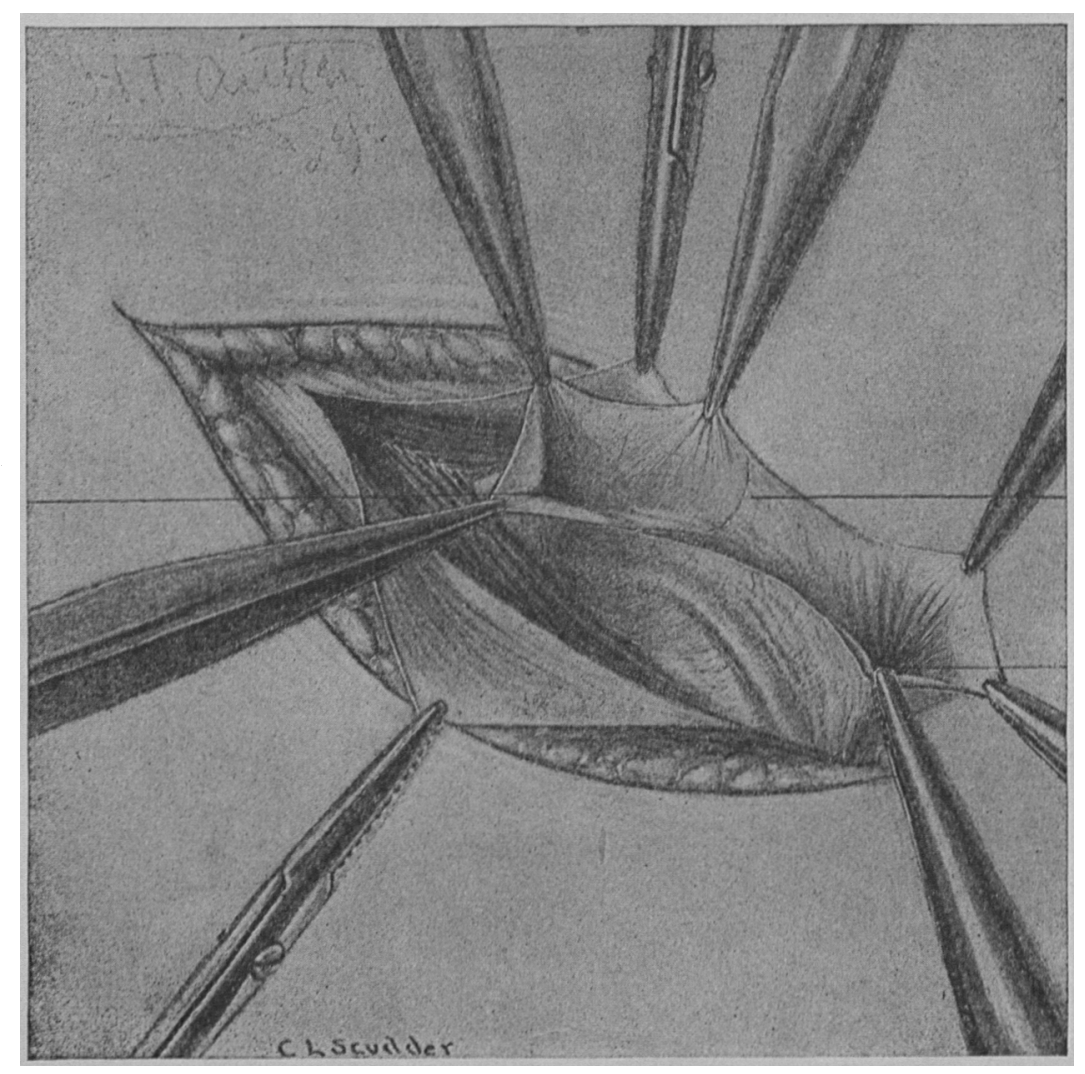

Frg. 4. The sac of the hernia has been opened. Note the interior of the sac leading towards peritoneal cavity above and to the scrotum below. Note the cord posteriorly to the sac and the dotted line across the sac indicating the line of section for further isolation of the peritoneum.

have seldom found it necessary to excise the veins of the cord. I have occasionally in children not transplanted the cord. I have utilized the fascia of the rectus abdominis a few times to reinforce the abdominal wall above the inner end of Poupart's ligament, as described by Halstead.

\section{RESULTS FOLLOWING OPERATION FOR HERNIA.}

I wish to report 244 operations done by me for herniæ of all kinds. There have been no deaths following operation in uncomplicated cases. The only deaths were in the strangulated group. There were 25 cases of strangulated hernia; 10 of these died; 3 were inguinal, 1 was ventral, 1 was femoral, and 5 of the 10 fatal cases were are comparatively few recurrences following the
There were 27 cases of ventral hernia; 11 did not report; 1 of the cases recurred; 1 died of strangulation; 14 of the cases in whom the end result was known were all right. That is, $93 \frac{1}{3} \%$ of the cases reported were cured.

There were $\cdot 13$ umbilical cases. There was no report from 3 cases. Of the remainder, 5 died of strangulation; 5 had no recurrence.

There were 5 epigastric herniæ. All of these reported, and each case is well.

There were 2 congenital cases, each of which died. The ages of the deaths of these 2 congenital cases were four hours and twenty-four hours respectively.

It will thus be seen after a careful analysis of 


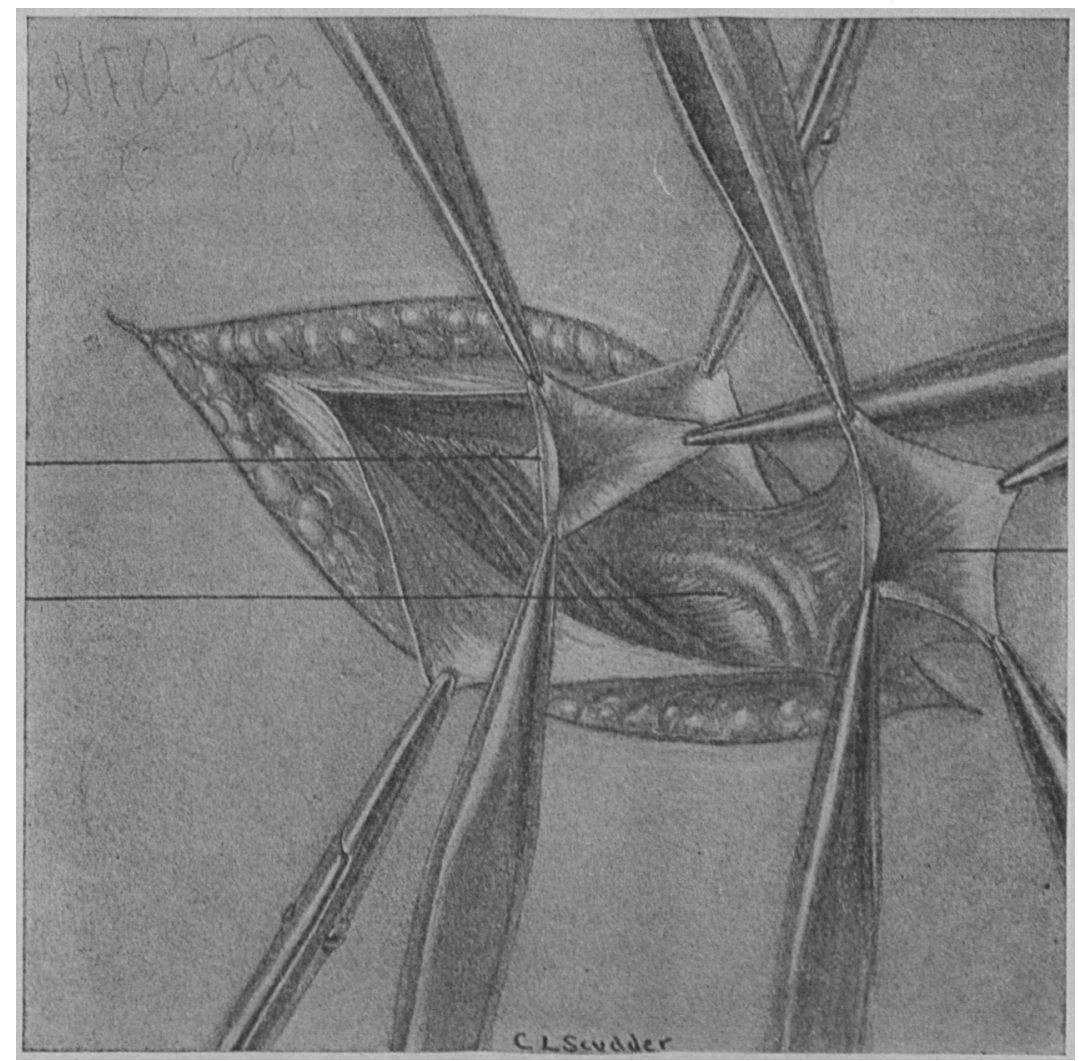

Fia. 5. The peritoneal portion of the hernial sac has been completely isolated; likewise the scrotal portion. Traction towards the symphysis pubis upon the peritoneal upper part of the sac facilitates proper placing of the sutures.

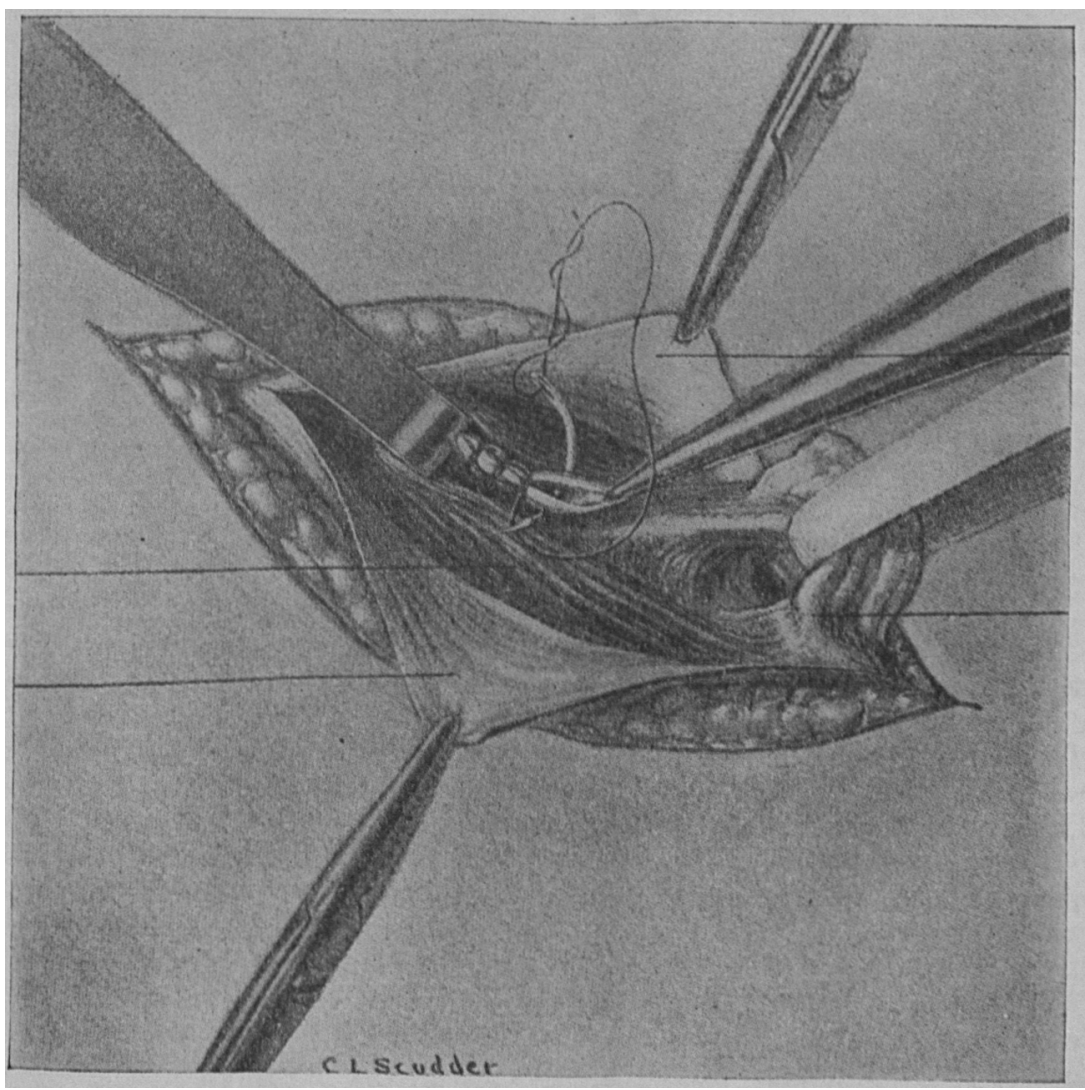

Fra. 6. The suture is being taken through and across the neck of the sac. Note retractor keeping internal ring region well in view. Note lifting of cord by gauze tape.

The Boston Medical and Surgical Journal as published by 
procedures employed. This investigation into the arise following any surgical operative procedure. condition of these hernia cases was undertaken There is, of course, a risk from the anesthetic. primarily to determine whether any material There is a risk from possible pneumonia. There change might be necessary in the technic of the is a risk from possible embolism or from sepsis, operation to secure better results. In analyzing but these risks must be taken and are to be the 10 cases in which recurrence is known to have disregarded under modern careful technic in occurred in the inguinal hernia group, conditions the question of a lesion so important as a hernia. were present which precluded, perhaps, the possi- There are a number of hernia operations bility of cure. In certain (2) of these cases a described, but if each is carefully analyzed it chronic cough which had persisted for years will be found that all of the satisfactory procontributed to the recurrence. One case was a cedures are based upon the same fundamental strangulated case, and it is very likely that the principles. Every operation for hernia preoperation was done with such haste on account supposes the existence of a pouch of peritoneum of the condition of the patient that the technic into which the abdominal viscus protrudes. was not as satisfactory as it might have been. The presence of this peritoneal pouch is a conIn the other 7 cases there is no explanation stant invitation to the contained viscus, whether apparent for the recurrence.

it be intestine or other organ, to protrude. Until

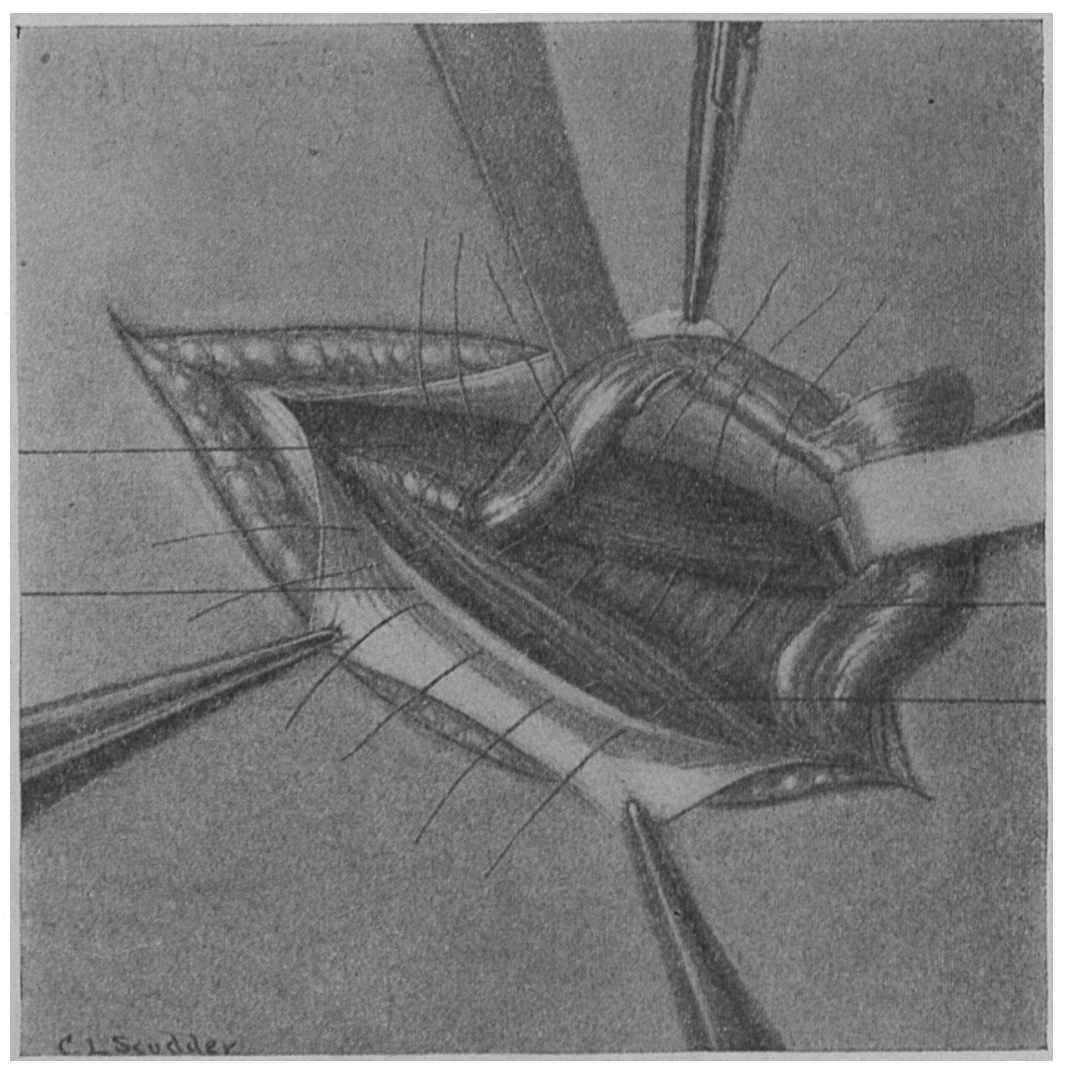

FIg. 7. Cord well raised, with all cord structures, from inguina! canal. Note suture placed beneath cord passing through conjoined tendon cremaster and Poupart's ligament. Note separation of inner part of divided fascia of external oblique.

I think it is fair to say that the operation this pouch is completely obliterated, the hernia described above as the Bassini operation for cannot be cured. The Bassini operation as inguinal hernia is most satisfactory and that described above seeks to obliterate the hernial if carefully performed gives at least a cure in peritoneal pouch on a level with the anterior $90 \%$ of the cases and possibly in $95 \%$ (Coley) abdominal wall so as to efface all evidence of a of the cases. There was no mortality attending pouch.

these operations for hernia. Certain of the After the peritoneal pouch is eliminated, the strangulated cases recovered. Ten strangulated further steps in the technic of any good hernial cases died.

CERTAIN FACTS OF IMPORTANCE.

operation consist in a firm closure of the muscular and fascial abdominal wall. The method employed in the operations here reported was that described above.

One should consider in connection with the question of mortality, and when advising an

The spermatic cord in the adult is best treated operation for hernia, the accidents which may by transplanting it to beneath the layer of the 


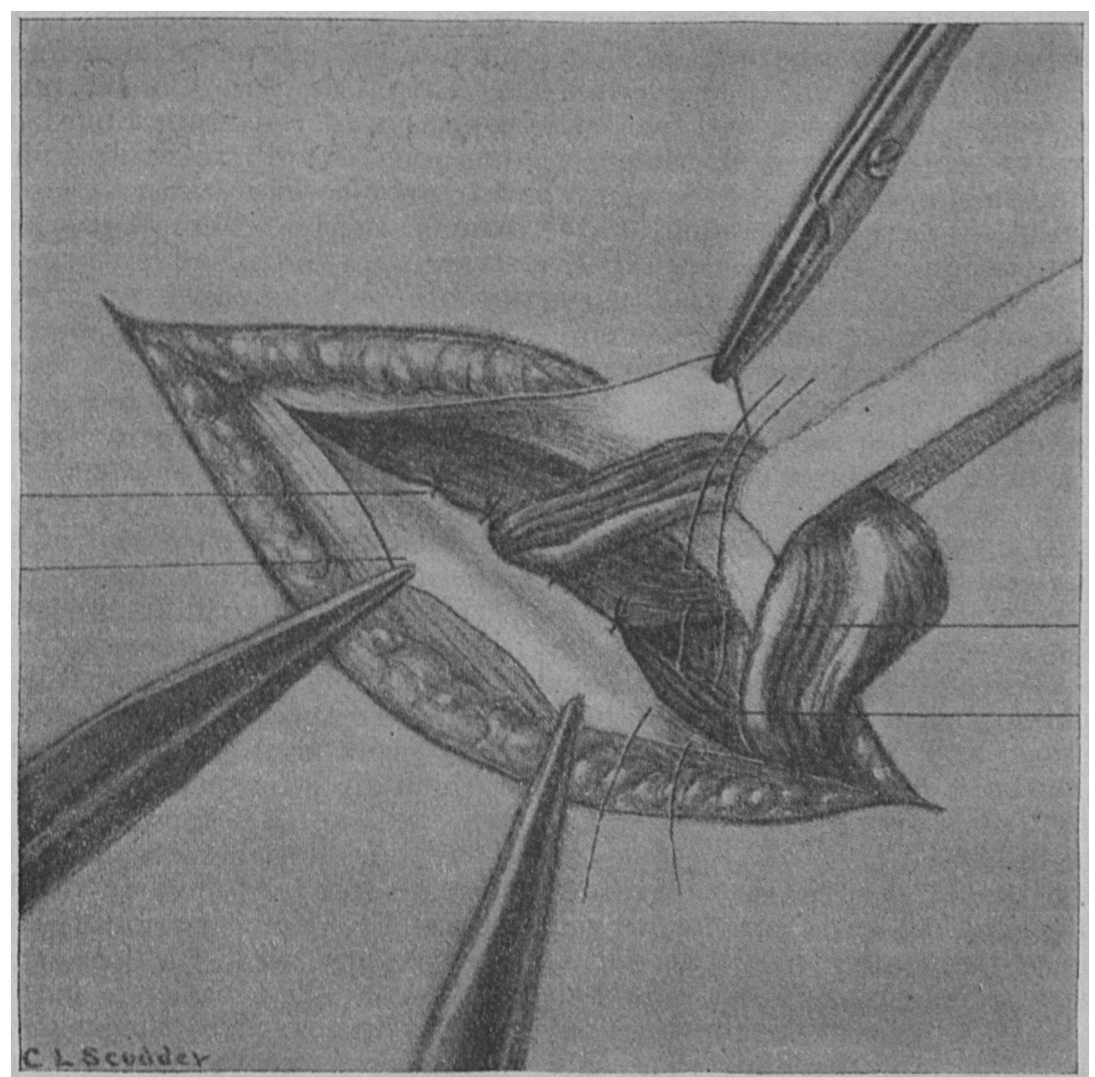

Fia. 8. Sutures above and below the cord tied excepting two.

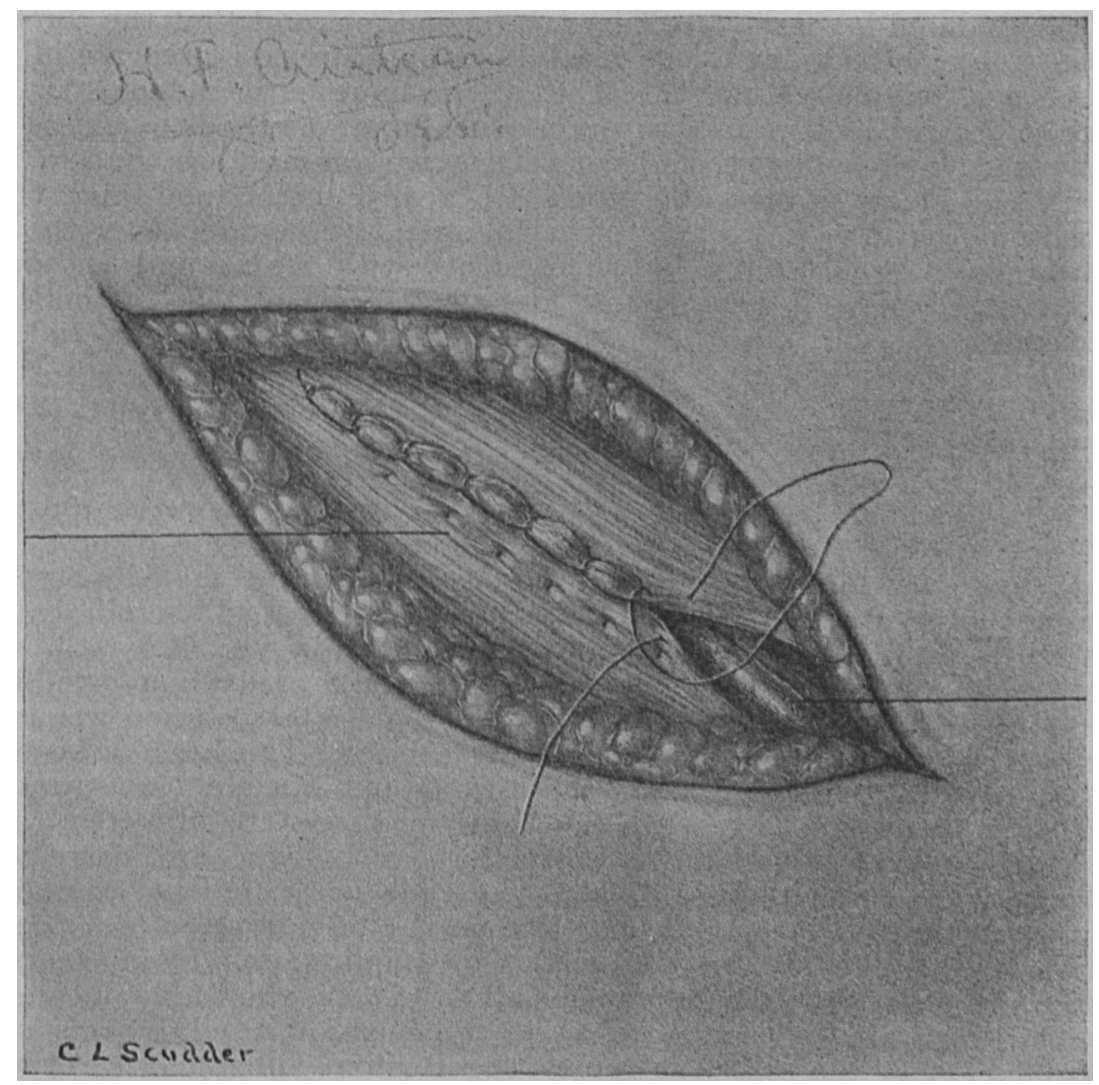

Fra. 9. Suture of the external oblique fascia above the cord.

The Boston Medical and Surgical Journal as published by

The New England Journal of Medicine. Downloaded from nejm org at WEST VIRGINIA UNIVERSITY LIBRARIES on September 16, 2016 For personal use only. No other uses without permission. From the NEJM Archive. Copyright $\odot 2010$ Massachusetts Medical Society. 
external oblique. In children it does not seem necessary to transplant the spermatic cord. In certain cases in adults I have not transplanted the cord and the results have been satisfactory.

There are very few cases indeed in which a resection of the spermatic veins is a necessary step in the operation for hernia. I should state the necessity for operation in this way: If there exists in any case of hernia a good-sized varicocele, it would be wise to remove these veins not only for the cure of the varicocele, but to obtain greater security in the wound.

If there is present upon the side of the hernia an undescended testicle, I believe that, unless there is some contra-indication, the removal of the testicle with ligation of the cord upon a level with the parietal peritoneum is indicated.

I believe that there are few cases in which the general anesthesia secured by ether will not be satisfactory. Local cocaine and spinal anesthesia should be reserved for cases too ill to be etherized, and for the aged and those with a tendency to chronic bronchitis. In certain strangulated cases where the patient is very ill a local or spinal anesthesia may be indicated.

It seems to me that it is unwise to operate upon infants under three years of age for hernia, and in the very aged operation is often contraindicated. These two extremes of life offer the chief exceptions to the operation for inguinal hernia. The operation for hernia is a safe operation.

It seems to me very important to emphasize the fact that an immediate operation should be done when a strangulated hernia is recognized. No taxis, not even the slightest, should be used in the presence of a strangulated hernia. The danger of damage to the gut and even of rupture of the gut and the danger of reducing the hernia en bloc is considerable. Delay in operating upon a strangulated hernia is unwise. Taxis of a strangulated hernia may be attended by such dire consequences that I believe it should almost never be employed.

\section{EDUCATIONAL METHODS IN THE ANTI- TUBERCULOSIS CAMPAIGN.*}

BY JOHN B. HAWES, 2D, M.D., BOston.

Tire two most important factors in the campaign against tuberculosis are:

(1) The isolation of the advanced consumptive, thus preventing the spread of the disease, and

(2) The education of the public and of the medical profession in regard to the nature of the disease, thus bringing about its early detection and treatment. This at least is the attitude of Massachusetts toward the tuberculosis problem.

The new sanatoria recently opened at North Reading, Lakeville and Westfield are primarily for the segregation and isolation of the advanced consumptive.

The medical profession of the state, as repre-

* Read before The Massachusetts Medical Society, June 7, 1910. sented by these Associated Committees, in conjunction with the Massachusetts Commission on Hospitals for Consumptives, the Health Inspectors of the State Board of Health and the numerous local anti-tuberculosis associations, are carrying on a campaign of education. The various methods employed, and the relative advantages and disadvantages of each, it is the purpose of this paper to discuss.

For many years we have been trying to educate the public in regard to tuberculosis. Ten or fifteen years ago, however, it was only the consumptive public whom we tried to educate, and that only in a desultory way. At that time, at any out-patient department, as, for example, at the out-patient department of the Massachusetts General Hospital, a consumptive coming for advice was told to take the examination for the Rutland State Sanatorium. If for any reason he was unable to do this, or if he was not accepted at this institution, he was handed a tract elaborately printed in whatever language he spoke, in which directions were given as to how he should live. "Take this tract," said the doctor, "put it on your bureau or wall at home, read it and learn it, and by following its directions you will get well." I am very doubtful if this method did much or any good. Indeed, I think it did harm in that it filled patients with a false sense of security in regard to themselves and the disease. Education to really educate must be more effective than this. One can easily prescribe an appropriate drug for a given ailment, but unless one sees that it is given in the proper doses, at the proper time, and that the directions in regard to its administration are carefully followed out, it will do more harm than good. It is the same with educational methods - unless we see that the facts we present are made effective, that they are really understood and that directions are followed, we could plaster the walls of our tenements with tracts and accomplish nothing. I shall describe certain methods which have been found effective for adults and for children.

\section{Educational Methods Suitable for Adults.}

(a) THE TUBERCULOSIS EXHIBIT.

This was perhaps the most effective method of education at our disposal. In this state I believe that it has done its best work and that the time of its greatest usefulness is past. In certain localities, however, it is still very valuable.

The large traveling exhibit of the Boston Tuberculosis Association was invaluable in arousing interest. This has now been fairly well aroused throughout Massachusetts, and our present energies may well be directed toward guiding the efforts of all who are interested and anxious to do some work for the campaign. These efforts must be largely spent in educating the ignorant, who require individual teaching rather than what can be shown by means of large exhibits. The exhibit used in this state, managed by the Boston Tuberculosis Association, is now in storage, where it will probably be kept for a year 